\title{
Laser Cleaning of Silicon Wafers: Mechanisms and Efficiencies
}

\author{
M. MOSBACHER ${ }^{1,2}$, M. BERTSCH ${ }^{1}$, H.-J. MÜNZER ${ }^{1}$, V. DOBLER ${ }^{1}$, B.-U. RUNGE ${ }^{1}$, \\ D. BÄUERLE ${ }^{2}$, J. BONEBERG ${ }^{1}$, P. LEIDERER ${ }^{1}$ \\ ${ }^{I}$ Universität Konstanz, Fachbereich Physik und Optikzentrum, Fach M676, D-78547 Konstanz, \\ Germany \\ ${ }^{2}$ Johannes - Kepler - Universität, Institut für Angewandte Physik, A-4040 Linz, Austria \\ E-mail: mario.mosbacher@uni-konstanz.de
}

\begin{abstract}
We report on experiments on the underlying physical mechanisms in the Dry- (DLC) and Steam Laser Cleaning (SLC) process. Using a frequency doubled, Q-switched Nd:YAG laser (FWHM=8 ns) we removed polystyrene (PS) particles with diameters from 110-2000 nm from industrial silicon wafers by the DLC process. The experiments have been carried out both in ambient conditions as well as in high vacuum $\left(10^{-6} \mathrm{mbar}\right)$ and the cleaned areas have been characterized by atomic force microscopy for damage inspection. Besides the determining the cleaning thresholds in laser fluence for a large interval of particle sizes we could show that particle removal in DLC is due to a combination of at least three effects: thermal substrate expansion, local substrate ablation due to field enhancement at the particle and explosive evaporation of adsorbed humidity from the air. Which effect dominates the process is subject to the boundary conditions. For our laser parameters no damage free DLC was possible, i.e. whenever a particle was removed by DLC we damaged the substrate by local field enhancement. In our SLC experiments we determined the amount of superheating of a liquid layer adjacent to surfaces with controlled roughness that is necessary for bubble nucleation. On smooth surfaces high superheatings were necessary, in good agreement with theoretical predictions. Rough surfaces exhibited only a much smaller superheating.
\end{abstract}

Keywords: Laser cleaning, cleaning mechanisms, field enhancement, superheating.

\section{Introduction}

In the production of integrated circuits one major source for production losses and malfunctions of the devices is the contamination of the silicon substrates by particles. Against the background of further shrinking linewidths of ICs (130 $\mathrm{nm}$ by the end of this year) it is clear that a fundamental requirement for the success in this field is the availability of a cleaning method capable of removing particles much smaller than $100 \mathrm{~nm}$ in diameter. Although traditional cleaning methods like ultra- and megasonics or brushing are continuously improved, it is not clear so far whether they will meet future demands in the semiconductor industry.

One of the new cleaning methods discussed as a possible complement to already existing procedures is laser cleaning. In Dry Laser Cleaning (DLC) [1-3] the surface to be cleaned is irradiated by a short laser pulse. In Steam Laser Cleaning (SLC) $[1,2,4]$ prior to the application of the laser pulse a liquid, e.g. a water-alcohol mixture, is condensed onto the surface.

Although laser cleaning is known for more than ten years now, still many questions related to the underlying physical processes are not answered. The authors of the first publications on the subject [1-4] suggested simple physical scenarios that were accepted for the interpretation of experimental data thereafter and were taken as a basis for theoretical modeling. Almost all authors explained DLC solely by the thermal expansion of the substrate and the adhering particle, SLC by explosive evaporation of the liquid condensed onto the surface.

Several models to describe these two processes were proposed [1,2,4-19], yet it is still not clear whether they accurately reflect the actual particle removal process for all laser parameters and cleaning environments studied so far. This is because they lack from major drawbacks. In DLC other possible cleaning mechanisms such as local ablation due to enhancement of the incoming laser intensity in the near field of the particle or evaporation of adsorbed humidity from the ambient air were not taken into account. However, first indications [17,19-23] report that both processes contribute to the particle removal. Models related to SLC are based very often on far-reaching assumptions concerning the bubble nucleation and growth process. Although there were investigations on this field in the past [24-26] they were carried out on rough substrates such as silver films, but not on smooth silicon wafers. Therefore the straightforward 
transfer of these results, e.g. superheating temperatures of the liquid, to SLC models of smooth surfaces is problematic and may lead to wrong results. In this paper we present the results of systematic experiments in DLC and SLC where. We determined in DLC the cleaning thresholds for a range of particle sizes from 110-2000 nm. Performing these experiments as well in ambient air as in high vacuum and carefully analyzing the cleaned surface after the process by an AFM, we investigated the cleaning mechanisms underlying the process. In SLC we could determine the superheating temperature of water for laser induced bubble nucleation on a silicon wafer.

\section{Experimental details}

For DLC we determined the removal efficiency and the cleaning thresholds in laser fluence for contaminants of different sizes and materials. We deposited individual spherical colloidal polystyrene $^{1}$ (PS) on industrial silicon wafers in a spin coating process described in [27]. Prior to particle application the wafers were cleaned in an ultrasonic bath in isopropanol (IPA). The samples prepared in this way were irradiated by a single Nd:YAG laser pulse $a=532 \mathrm{~nm}$, $\mathrm{FWHM}=8 \mathrm{~ns}$ ) either in ambient conditions (relative humidity $30-40 \%$ ) or in high vacuum ( $\mathrm{HV}, 10^{-6}$ mbar). In ambient cleaning a flow of pressurized, filtered air was used to blow away the removed particles and to prevent their redeposition.

Under ambient conditions particle removal in the cleaned area (about $1 \mathrm{~mm}^{2}$ ) was detected by a light scattering technique [28]. A $5 \mathrm{~mW}$ HeNe laser illuminated a spot with a diameter of $0.5 \mathrm{~mm}$, which corresponds to several hundred particles monitored, its scattered light was detected by a photomultiplier. The monitored area was much smaller than the illuminated area, therefore in this case the laser fluence can be considered as almost homogeneous. In $\mathrm{HV}$ we determined the fluences necessary for particle removal by inspecting the illuminated spot with an optical microscope prior and after the laser pulse. We measured the threshold cleaning fluence relatively to the melting threshold of $\mathrm{Si}$, monitoring the reflected light of the HeNe laser with ns time resolution. As the laser fluence for the onset of melting of silicon is well known, this can be used for a conversion of relative fluences into absolute numbers.

Figure 1 shows the setup for the bubble nucleation experiments. A Q-switched Nd:YAG laser $(\lambda=532 \mathrm{~nm}$, FWHM $=8 \mathrm{~ns}$ ) heated the silicon sample. A cw Ar-ion laser $(\lambda=488 \mathrm{~nm})$ was focused onto the sample, and its light, scattered by the nucleated bubbles, was collected in forward direction and perpendicular to the incident ray. Sample and liquid were placed in a fused silica cuvette that could be

\footnotetext{
${ }^{1}$ IDC, Portland, Oregon, USA
}

heated up to $360 \mathrm{~K}$. Using a pinhole, which allowed only the center part of the beam to pass (where the laser fluence is

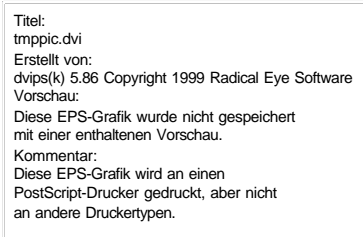

Fig. 1 Experimental setup for the bubble nucleation experiments. (P)BS: (polarizing) beam split, PD: photo diode, IF: interference filter $(488 \mathrm{~nm}), \mathrm{M}$ : mirror, AFR: achromatic Frensel-rhombus, L: lens, NFG: neutral glas filter, FM: laser fluence meter (Field Master, COHERENT). The polarization of

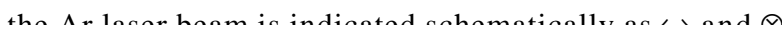

quite uniform), we derived the laser fluence from the measured transmitted light pulse and the known pinhole area.

\section{DLC in ambient conditions}

A first step in the investigation of DLC is the study of particle removal in ambient conditions. This environment represents the conditions that may be found in a possible future application of the process.

In Fig. 2 the thresholds in applied laser fluence for the removal of PS particles are plotted as a function of the particle size. From this diagram one can obtain a lot of information on the process.

First we would like to bring to mind two very imp ortant thresholds in the laser cleaning process. One of them is the threshold for the onset of melting of the bare substrate. As DLC is aimed for an industrial application, any change of the structure of the silicon wafer, i.e. the silicon substrate and a native oxide layer of specified thickness, as induced by melting has to be strictly avoided. From experiments [29-31] this melting threshold is known to be about $280 \mathrm{~mJ} / \mathrm{cm}^{2}$, which therefore represents the upper limit of applicable laser fluence. The second threshold, also indicated in Fig. 2, is the cleaning threshold of the SLC process. In previous investigations [28] we found this threshold to be independent of the particle diameter $(60-800 \mathrm{~nm})$ and material (PS, $\mathrm{SiO}_{2}, \mathrm{Al}_{2} \mathrm{O}_{3}$ ). 
In order to obtain information on the dependency of the cleaning threshold on the particle size we investigated many different particle diameters in the range of 110-2000 nm. It turned out that only by the investigation of this large variety

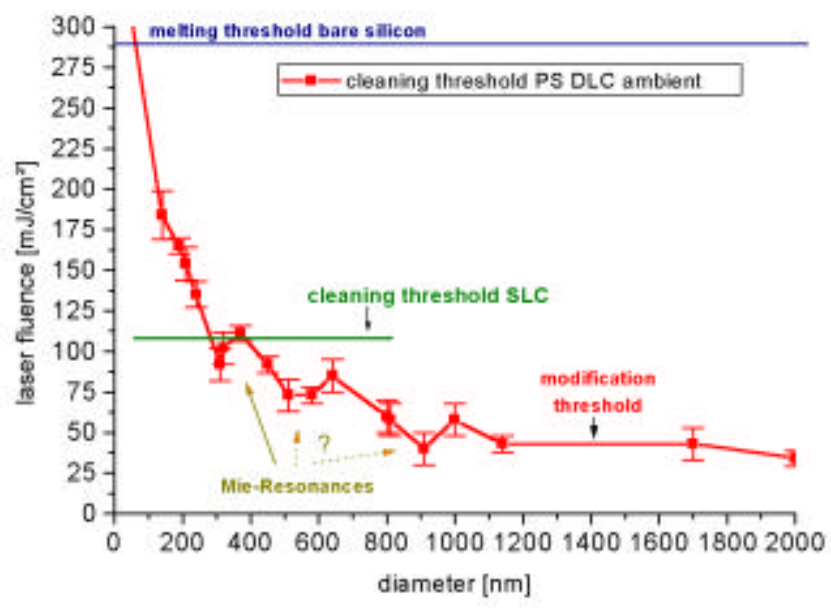

Fig. 2 Threshold in the applied laser fluence for particle removal in DLC in ambient air. Particles smaller in diameter than $110 \mathrm{~nm}$ could not be removed.

of particle sizes some of the main characteristics of the dependence of the cleaning threshold on the particle diameter could be revealed.

At first sight the shape of the curve follows a $1 / \mathrm{r}^{\mathrm{k}}-$ trend, where $\mathrm{r}$ denotes the particle radius and $1<\mathrm{k}<2$. This monotonic behavior was predicted by the DLC models cited above, and in fact already the first publications on DLC reported that smaller particles are harder to remove than larger ones [1-3]. If one looks closely, however, one discovers an oscillating behavior of the threshold fluences, not predicted by the models. This is illustrated in Fig. 2 by the line connecting the data points.

The origin of these oscillations is given by the enhancement of the incoming laser field in the near field of the particle, a phenomenon described by a modified Mie theory for the geometry of particles on the surface. In Mie theory, particles illuminated by a plain electromagnetic wave, act as a cavity for the incoming beam and enhance the incoming intensity at their light averted side. This is illustrated in Fig. 3. For some values of the size parameter $\pi \mathrm{d} / \lambda$ this enhancement is particularly effective. Resonances influencing the cleaning threshold are obtained. This results in the oscillatory behavior of the cleaning threshold fluence as function of the particle diameter. However, it should be pointed out, that the line connecting the data points is just a guide to the eye and does probably not describe the field enhancement efficiency as function of the particle diameter.

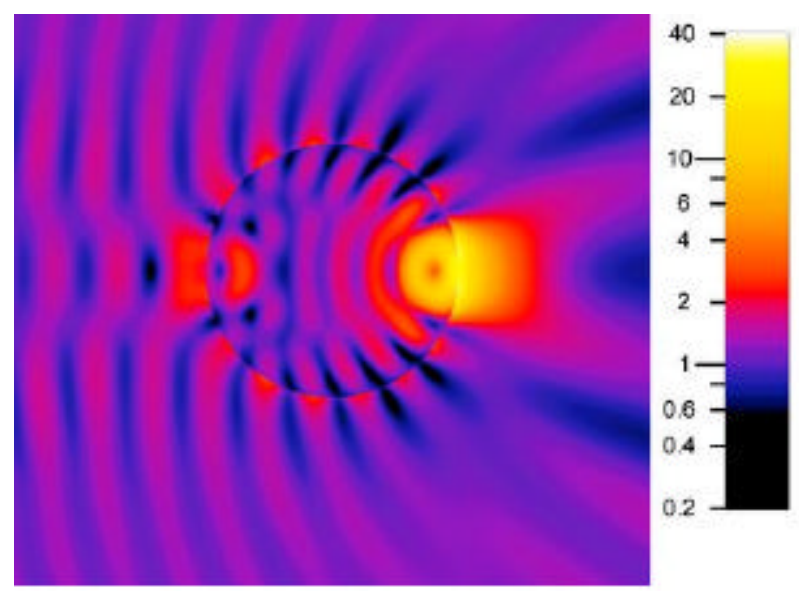

Fig. 3 Intensity $\left(E^{2}\right)$ of the electromagnetic field around a PS particle (diameter $1700 \mathrm{~nm}$, refractive index 1.59) in vacuum when illuminated by a laser at $\lambda=800 \mathrm{~nm}$. The laser enters from the left, the light is polarized in the image plane. At the light averted side of the particle the intensity is enhanced by The number of particle sizes used in our experiments is not sufficient to resolve this dependency. Nevertheless the resonances account for the deviation of the curve from a smooth, monotonic shape.

Clearly, such an enhanced laser fluence underneath particles adhering to a surface has an important impact on the laser cleaning process, first results have been reported in $[22,23]$. It decreases the applied laser fluence necessary to remove particles and is responsible for surface damage by local melting/ablation of the substrate. Depending on the pulse length used for the cleaning laser, and hence the thermal diffusion length during this laser pulse, local ablation leads either to the formation of steep holes (ultrashort pulses $<100 \mathrm{ps}$ ) or shallow melting/ablation sites (nanosecond pulses). Examples for the case of ns pulses are shown in Fig. 4.

In DLC using fs pulses the removal of a particle is always accompanied by the formation of a hole, i.e. the hole formation threshold is identical with cleaning threshold. From this one can conclude that for these pulse lengths the particles are removed by the momentum transfer of the ablated substrate material rather than by thermal expansion local ablation acts as a cleaning mechanism [22]. This shows very clearly that for a damage free DLC ultrashort pulses are not suitable. On the other hand the shape of the local ablation areas reflects the field distribution underneath the particle [32] and can therefore be used to experimentally

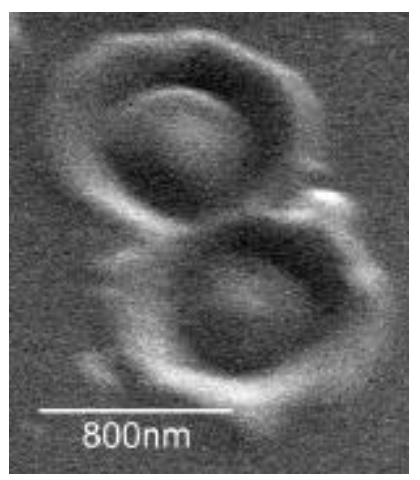

Fig. 4 Scanning electron microscope image of a typical melting site induced by field enhancement after ns 
investigate the field structure in the particles' near field. Together and in comparison with numerical calculations this helps to understand these fundamental physical processes underlying laser cleaning.

Against the background of field enhancement as origin of surface damage, it is a natural question to determine not only the cleaning threshold fluences in DLC, but also the local melting/ablation thresholds. The latter, instead of the melting threshold of the bare silicon surface, represent the true upper limit for the applicable laser fluence and are by their nature particle dependent. For its determination we made use of the Gaussian spatial beam profile of our cleaning laser. Due to this profile a spatial variation in the cleaned area corresponds to a variation in the locally applied laser fluence. In a post process analysis we investigated the cleaned areas of our samples with an AFM. By this method we imaged the field enhancement induced damage sites (cf. Fig. 4 for an SEM image), a typical example can be seen in Fig. 5.

Imaging damage sites at different locations in the cleaned areas and especially at their borders, which correspond to the cleaning threshold fluence, we determined the damage threshold for each particle size. For all particles investigated the cleaning threshold was identical with the damage threshold. Damage free DLC was not possible applying the laser parameters we used!

The AFM images contain evan more information on the particle removal mechanism as they reveal quantitative topographic information. All the investigated damage sites showed the same features: a "trench" surrounded a central "hillock". Generally spoken the hillock was lower at high laser fluences and the trench deeper, for low laser fluences a hillock was detectable but the trench almost disappeared. In

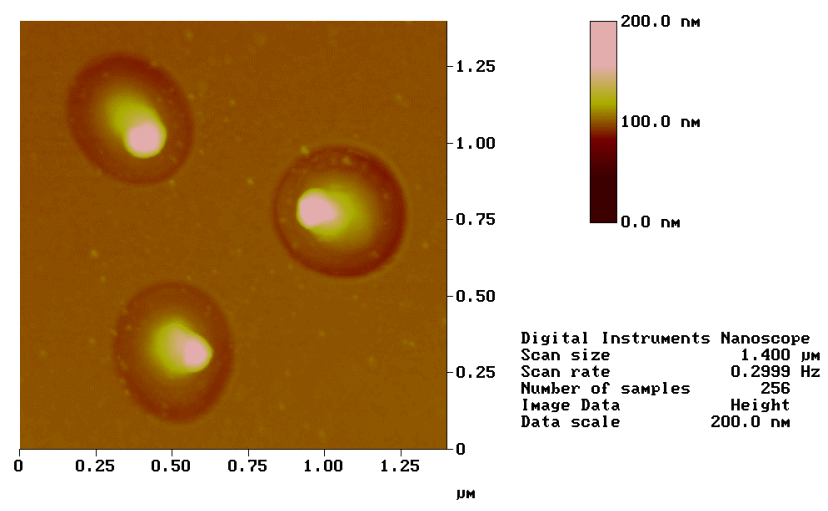

050900.014

Fig. 5 Atomic force microscopy (AFM) image of a damage site after DLC using ns pulses. The damage site was created by an agglomerate of three PS spheres $800 \mathrm{~nm}$ in diameter. Note the typical damage morphology: a shallow trench

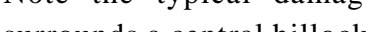

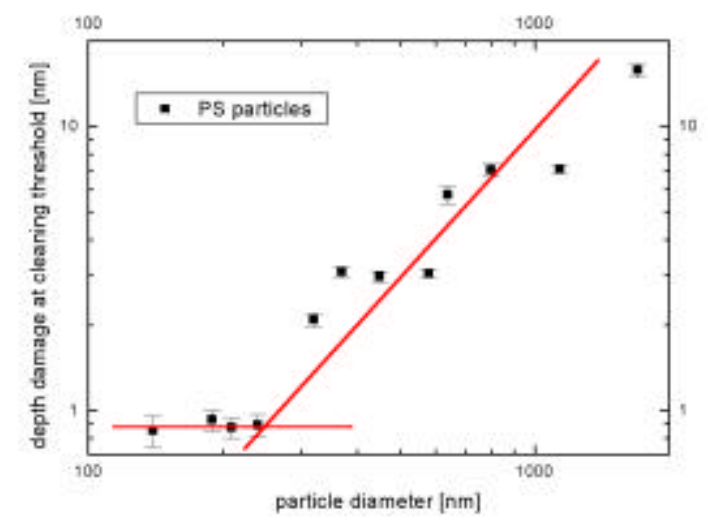

Fig. 6 Trench depth of the damage sites as a function of the particle size when the particles were removed in DLC applying the threshold cleaning fluence. For particles larger than $250 \mathrm{~nm}$ in diameter the depth increases strongly with

Fig. 6 we plotted in a double logarithmic graph the mean trench depths for the investigated particles for damage that occurred at the cleaning/damage threshold.

This plot clearly shows two regimes: for particles smaller than about $250 \mathrm{~nm}$ in diameter the depth remains almost constant at about $1 \mathrm{~nm}$. For larger particles we found a strong increase in the trench depth, the volume of the hillock was smaller than that of the trench - ablation had taken place.

From this observation we conclude, that even for DLC using ns pulses local ablation of the substrate plays a role as cleaning mechanism for "large" particles where the field enhancement is higher and thus provides fluences high enough for ablation. At smaller particles field enhancement probably causes local melting, but no ablation.

\section{DLC in vacuum}

Several authors report that in their DLC experiments an increase in ambient humidity led to an increase in cleaning efficiency $[17,19,21]$. This was attributed to the additional cleaning force provided by the explosive evaporation of the water adsorbed at the interstice between particle and surface. In a recent publication on the DLC of polyimide [19] the authors systematically increased the humidity from $30 \%$ to $90 \%$ and could find an efficiency increase - a strong support for the above interpretation. However, these experiments were not carried out on silicon and the obtained data originate only from two particle sizes.

In our experiments we chose the complementary approach to demonstrate the influence of the ambient humidity. We repeated the experiments described in Sec. 3 in high vacuum and decreased the amount of adsorbed water 
by allowing the samples to degas approximately 10 hours at $10^{-6}$ mbar. The results and the comparison with the ambient

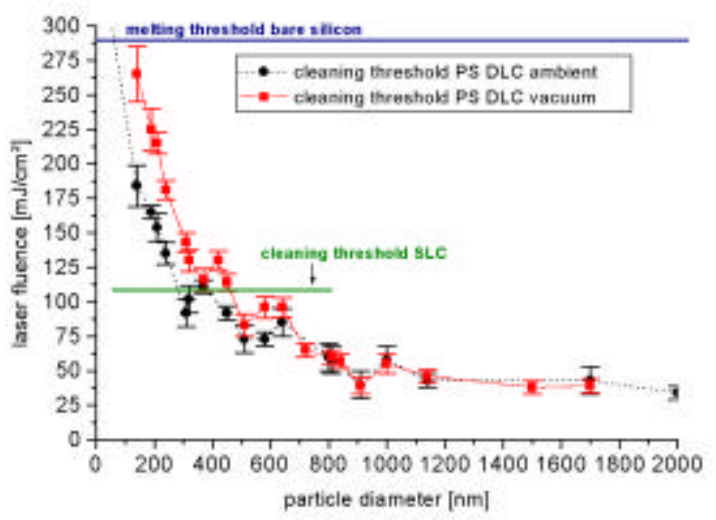

Fig. 7 Comparison of the cleaning thresholds in ambient air and high vacuum. In vacuum the thresholds are distinctly higher.

values are presented in Fig. 7. In vacuum the laser fluences necessary for particle removal have been higher for particles smaller than about $800 \mathrm{~nm}$ in diameter. For larger particles no difference in the threshold could be detected between ambient conditions and HV.

We attribute this to the action of different predominant cleaning mechanisms for different particle sizes. Cleaning in ambient conditions is facilitated by the explosive evaporation of adsorbed humidity from air. When exposed to vacuum for several hours the amount of humidity at the particle-surface contact is significantly decreased, the contribution of explosive evaporation to the cleaning forces decreases, and consequently the cleaning threshold increases. This argumentation should also be valid for particles larger than $800 \mathrm{~nm}$ in diameter. However, as discussed above, for large particles field enhancement induced local ablation strongly contributes to the cleaning process. And of course this is the case as well in ambient air as in vacuum and no large difference in the cleaning thresholds of the two environments is detected.

\section{Laser induced bubble nucleation in SLC}

Laser cleaning can not only be promoted by the adsorption of atmospheric humidity, but also by the condensation of a liquid onto the sample by purpose. This process, called Steam Laser Cleaning, relies very much on the explosive evaporation of this liquid. For this reason the investigation of pulse laser induced bubble nucleation and growth at liquid/solid interfaces provides the key for the understanding of the process and the increase of its efficiency.
Previous work on the nucleation of bubbles on metallic films in SLC [24-26] discovered that quite moderate superheatings of about $20 \mathrm{~K}$ are sufficient for their generation, a value much lower than the theoretically predicted one of $200 \mathrm{~K}$ for water [33]. This has been attributed to the surface roughness of these substrates. But to our knowledge there are no systematic studies that confirm this roughness-dependency of the threshold.

We therefore studied the nucleation of bubbles at a superheated liquid/solid interface under controlled surface roughness using the setup described in Sec. 2. Scattered light was observed in both directions when the incident laser fluence reached a well defined threshold. This indicates a sharp nucleation threshold. The threshold decreases with increasing starting temperature $\mathrm{T}_{0}$ as less energy is needed to reach the nucleation temperature. Figure 8 shows these temperature dependent thresholds for the examined systems. The lines are linear extrapolations to vanishing fluence and yield the nucleation temperature by their intersection with the temperature axis.

In a first series of experiments we determined the nucleation thresholds and superheating temperatures for water on a $50 \mathrm{~nm}$ silver film thermally evaporated on glass. Here we found a nucleation temperature of $(130 \pm 30)^{\circ} \mathrm{C}$, only slightly above the one reported in literature [24-26].

Water on atomically smooth silicon exhibited a nucleation temperature of $(250 \pm 30){ }^{\circ} \mathrm{C}$ in close agreement to the expected value from theory and far above that one measured on the silver film. To verify the influence of the surface roughness we structured a silicon wafer with holes (diameter approx. $500 \mathrm{~nm}$, depth approx. $40 \mathrm{~nm}$, density approx. $0.05 / \mu \mathrm{m}^{2}$, for the preparation method see reference [23]). On this substrate the nucleation temperature decreased strongly to $(160 \pm 13){ }^{\circ} \mathrm{C}$, close to that of the rough silver film - a clear evidence for the influence of surface roughness.

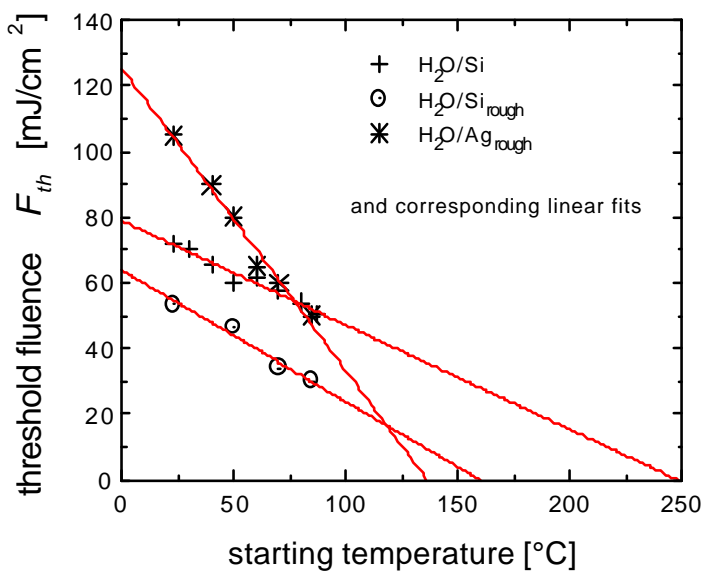

Fig 8 Comparison of the bubble nucleation thresholds and extrapolated superheating limits of water on substrates of different roughness. 


\section{Discussion and conclusion}

Our conclusions are of relevance both for a future application of laser cleaning as well as for the understanding of the underlying physical processes.

The determination of cleaning thresholds in DLC under ambient conditions confirmed a general "trend" already obtained by other authors $[1,2,15,17,19,34]$ and predicted by the models: cleaning thresholds for smaller particles tend to be higher than for larger ones. However, this is not a strict rule. In contrast to the above cited experiments we used a large variety of particle diameters ranging from $110 \mathrm{~nm}$ to $4100 \mathrm{~nm}$ and could show that the dependency of the cleaning threshold as function of the particle diameter is non-monotonous.

This is related to the enhancement of the incoming laser beam in the near field of the particles and the Mieresonances occurring at certain values of the size parameter. Field enhancement does have two important consequences. First, as it locally increases the laser fluence underneath the particle, it decreases the incident laser fluence necessary for particle removal. This was also applied by the authors of $[15,20,35]$ to explain differences in the theoretically predicted and actually measured cleaning threshold fluences.

Yet there is a second obvious consequence: a locally enhanced laser intensity drastically lowers the threshold for surface damage, and indeed we could observe surface damage either due to melting (small particles) or local substrate ablation (large particles) whenever a particle was removed in DLC. This substrate damage at incident laser fluences much lower than the melting threshold of the bare silicon surface was not reported before. Kane and Halfpenny [36] reported on the reduction of the ablation threshold of a glass substrate covered by $\mathrm{Al}_{2} \mathrm{O}_{3}$ particles all over the beam area, but our damage sites are localized directly beneath the former positions of the particles, the surface outside remains intact. The local substrate ablation does not only represent a mechanism of damage, but also contributes to the removal of the particles as cleaning mechanism.

At this point it is worth to mention that the observation of the size dependent Mie resonances in the threshold fluence was only possible due to the use of spherical colloidal particles with a small size distribution $( \pm 5 \%)$.

Performing the DLC experiments in HV and comparing the results to ambient cleaning we could show that for small particles humidity condensed at the particles from the air contributes to the particle removal as a third cleaning mechanism, similar to SLC. For large particles the cleaning force provided by the evaporating liquid can be neglected versus the one provided by local ablation - the cleaning thresholds are similar for HV and ambient cleaning.
With respect to applications the comparison of the cleaning thresholds in DLC and SLC is quite important and could be done for the first time on the basis of our systematic measurements. For particles smaller than about $400 \mathrm{~nm}$ in diameter - the most interesting sizes in the semiconductor industry - the cleaning thresholds of SLC are lower. At a first glance, DLC is the more appropriate method for larger particles as its cleaning thresholds are lower. However, due to field enhancement particle removal by DLC is always accompanied by surface damage. At our laser parameters $(\lambda=532 \mathrm{~nm}, \mathrm{FWHM}=8 \mathrm{~ns})$ DLC is not applicable for surface cleaning.

Regarding the modeling of DLC the consequences of our results are obvious. So far particle removal has always been ascribed to the thermal expansion of the substrate solely, field enhancement was only taken into account by Lukyanchuk et al. [35] as increased laser fluence, but not via the cleaning mechanism local ablation. The third cleaning mechanism, evaporation of adsorbed ambient humidity, is not incorporated into any model published so far. This is even more important, as most of the experiments compared to these theories were performed in ambient conditions [7,9$12,14,16,34]$. Only in [15] the experiments were conducted in vacuum and Vereecke et al. [17] varied the relative humidity and reported a higher cleaning efficiency at increased humidity levels.

Future models should incorporate all three cleaning mechanisms and treat DLC as an interplay of all of them. Depending on the process parameters (laser wavelength, pulse duration, optical constants of the materials etc.) their overall contribution to the cleaning will vary. It should also be pointed out that the cleaning mechanisms are not independent of each other, e.g. adsorbed humidity may influence the field enhancement pattern - further investigations are necessary in this respect.

Our investigation of bubble nucleation and superheating of water in SLC also provides important information on the cleaning process. In contrast to previous investigations on rough surfaces [24-26], where only small superheating of about $20^{\circ} \mathrm{C}$ was reported, we detected superheating values of about $150^{\circ} \mathrm{C}$ on smooth silicon wafers for the first time. The difference can clearly be attributed to the different roughnesses in the experiments.

In summary, we have shown that DLC is a more complex process than thought so far. Only particles larger than $100 \mathrm{~nm}$ in diameter could be removed. Due to local field enhancement any particle removal at the laser parameters used was always accompanied by surface damage which inhibits any practical application of the process for surfaces which must not be damaged by any means, like silicon wafers. These findings argue for the application of SLC in wafer cleaning as it is capable of removing smaller particles 
and as the cleaning threshold is independent of the particle properties.

Acknowledgments. We thank Prof. B. Luk'yanchuk (DSI, Singapore) and Dr. Nikita Arnold (Johannes-KeplerUniversity, Linz, Austria) for useful discussions. Financial support by EU TMR project "Laser Cleaning" $\left(\mathrm{n}^{\mathrm{o}}\right.$ ERBFMRXCT98 0188) and the Konstanz Center for Modern Optics is gratefully acknowledged. Wacker Siltronic supplied the industrial silicon wafers.

\section{References}

[1] W. Zapka, W. Ziemlich and A.C. Tam: Appl. Phys. Lett. 58 (1991) 2217.

[2] A. C. Tam, W. P. Leung, W. Zapke and W. Ziemlich: J. Appl. Phys. 71 (1992) 3515.

[3] A.C. Engelsberg: Proc. MRS 315 (1993) 255.

[4] K. Imen, J. Lee, S. D. Allen: Appl. Phys. Lett. 58 (1991) 203.

[5] J. D. Kelley and F. E. Hovis: Microelectronic Eng. 20 (1993) 159.

[6] J. M. Lee, K. G. Watkins, W. M. Steen: Appl. Phys. A 71 (2000) 671.

[7] Y. F. Lu, W. D. Song, M. H. Hong, B. S. Teo, T. C. Chong, T. S. Low: J. Appl. Phys. 80 (1996) 499.

[8] Y. F. Lu, W. D. Song, B. W. Ang, M. H. Hong, D. S. H. Chan, T. S. Low: Appl. Phys. A 65 (1997) 9.

[9] Y. F. Lu, W. D. Song, K. D. Ye, M. H. Hong, D. M. Liu, D. S. H. Chan, T. S. Low: Appl. Surf. Sci. 120 (1997) 317.

[10] Y. F. Lu, W. D. Song, Y. Zhang, T. S. Low: Proc. SPIE 3550 (1998) 7.

[11] Y. F. Lu, W. D. Song, T. S. Low: Materials Chemistry and Physics 54 (1998) 181.

[12] Y. F. Lu, W. D. Song, M. H. Hong, D. S. H. Chan, T. S. Low: Proc. SPIE 3097 (1997) 352.

[13] Y. F. Lu, Y. Zhang, Y. H. Wan, W. D. Song: Appl. Surf. Sci. 138-139 (1999) 140.

[14] Y. F. Lu, Y. W. Zheng, W. D. Song: Appl. Phys. A 68 (1999) 569.

[15] Y. F. Lu, Y. W. Zheng, W. D. Song: J. Appl. Phys. 87 (2000) 1534.

[16] W. D. Song, Y. F. Lu, K. D. Ye, C. K. Tee, M. H. Hong, D. M. Liu, T. S. Low: Proc. SPIE 3184 (1997) 158.

[17] G. Vereecke, E. Röhr, M. M. Heyns: J. Appl. Phys. 85 (1999) 3837.

[18] X. Wu, E. Sacher, M. Meunier: J. Appl. Phys. 87 (2000) 3618.

[19] T. Fourrier, G. Schrems, T. Mühlberger, J. Heitz, N. Arnold, D. Bäuerle, M. Mosbacher, J. Boneberg, P. Leiderer: Appl. Phys. A 72 (2001) 1.

[20] B. S. Lukyanchuk, Y. W. Zheng, Y. F. Lu: Proc. SPIE 4065 (2000) 576.
[21] P. Leiderer, J. Boneberg, V. Dobler, M. Mosbacher, H.-J. Münzer, N. Chaoui, J. Siegel, J. Solis, C. N. Afonso, T. Fourrier, G. Schrems, D. Bäuerle: Proc. SPIE 4065 (2000) 249.

[22] M. Mosbacher, H.-J- Münzer, J. Zimmermann, J. Solis, J. Boneberg, P. Leiderer: Appl. Phys. A 72 (2001) 41.

[23] H.-J. Münzer, M. Mosbacher, M. Bertsch, J. Zimmermann, P. Leiderer, J. Boneberg: J. Microscopy 202 (2001) 129.

[24] O. Yavas, P. Leiderer, H. K. Park, C. P. Grigoropoulos, C. C. Poon, W. P. Leung, N. Do, A. C. Tam: Phys. Rev. Lett. 70 (1993) 1830.

[25] H. K. Park et al: Appl. Phys. Lett. 68 (1996) 4072

[26] O. Yavas, A. Schilling, J. Bischof, J. Boneberg, P. Leiderer: Appl. Phys. A 64 (1997) 331.

[27] M. Mosbacher, H.-J. Münzer, M. Bertsch, V. Dobler, N. Chaoui, J. Siegel, R. Oltra, D. Bäuerle, J. Boneberg, P. Leiderer: Particles on Surfaces 7, K. L. Mittal (Ed.), VSP publishing (2001).

[28] M. Mosbacher, V. Dobler, J. Boneberg, P. Leiderer: Appl. Phys. A 70 (2000) 669.

[29] H. Kurz, L. A. Lompré, J. M. Liu: J. de Physique 10 (1983), C5.

[30] D. H. Lowndes, R. F. Wood, D. Westbrook: Appl. Phys. Lett 43 (1983) 258.

[31] J. Boneberg: Metallische Dünnfilmschmelzen von Halbleiterschichten nach ns-Laser-Annealing, $\mathrm{PhD}$ thesis, University of Konstanz (1996).

[32] H.-J. Münzer, M. Mosbacher, M. Bertsch, O. Dubbers, F. Burmeister, A. Pack, R. Wannemacher, B.-U. Runge, D. Bäuerle, J. Boneberg, P. Leiderer: this volume.

[33] C. T. Avesidian: J. Phys. Chem. Ref. Data 14 (1985) 695.

[34] D. R. Halfpenny, D. M. Kane: J. Appl. Phys. 86 (1999) 6641.

[35] B. S. Lukyanchuk, Y. W. Zheng, Y. F. Lu: Proc. SPIE. to be published.

[36] D. M. Kane, D. R. Halfpenny: J. Appl. Phys. 87 (2000) 4548 . 